\title{
Transatlantica
}

Revue d'études américaines. American Studies Journal

Jacques-Henri Coste et Vincent Dussol (dir.), The Fictions of American Capitalism: Working Fictions and the Economic Novel

\section{Alexia Blin}

\section{OpenEdition}

\section{Journals}

Édition électronique

URL : https://journals.openedition.org/transatlantica/15413

DOI : 10.4000/transatlantica. 15413

ISSN : $1765-2766$

Éditeur

Association française d'Etudes Américaines (AFEA)

Référence électronique

Alexia Blin, « Jacques-Henri Coste et Vincent Dussol (dir.), The Fictions of American Capitalism: Working Fictions and the Economic Novel », Transatlantica [En ligne], 1 | 2020, mis en ligne le 01 décembre 2020, consulté le 01 février 2023. URL : http://journals.openedition.org/transatlantica/15413 ; DOI : https:// doi.org/10.4000/transatlantica.15413

Ce document a été généré automatiquement le 1 février 2023

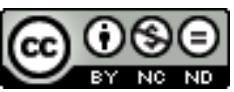

Creative Commons - Attribution - Pas d'Utilisation Commerciale - Pas de Modification 4.0 International - CC BY-NC-ND 4.0

https://creativecommons.org/licenses/by-nc-nd/4.0/ 


\title{
Jacques-Henri Coste et Vincent Dussol (dir.), The Fictions of American Capitalism: Working Fictions and the Economic Novel
}

\author{
Alexia Blin
}

\section{RÉFÉRENCE}

COSTE, Jacques-Henri, et Vincent DUSSOL dir. The Fictions of American Capitalism: Working Fictions and the Economic Novel. Cham: Palgrave MacMillan, 2020, 408 p., ISBN :

978-3-030-36563-9, 103,99€ (85,59€ pour l'e-book).

1 The Fictions of American Capitalism est un ouvrage collectif, issu de plusieurs journées d'études, qui réunit les contributions d'historien-nes, d'économistes, de sociologues et de spécialistes d'études littéraires états-uniennes. Malgré la diversité d'horizons des contributeurs et contributrices de l'ouvrage, celui-ci présente une certaine unité dans l'ambition disciplinaire qu'il revendique. En effet, le projet général du livre est de dépasser l'idée que "literature and economics make odd bedfellows " (p.336), pour s'intéresser précisément à la fécondité de leurs interactions. On pourrait résumer l'objectif du livre en disant qu'il cherche, à partir du cas des États-Unis, à répondre à deux questions : Que doit l'économie (et plus précisément le capitalisme) à la fiction? Et réciproquement, que doit la fiction à l'économie?

2 Ces deux questions sont formulées, notamment par les directeurs de l'ouvrage, JacquesHenri Coste et Vincent Dussol, sous la forme d'un appel à l'intention des économistes et des spécialistes d'études littéraires à décloisonner leur discipline respective. La stricte séparation qui existe aujourd'hui entre ces deux champs de recherche n'a pas toujours été la règle comme le font remarquer les auteurs. Elle s'est accentuée en particulier à partir des années 1970, dans le double sillage du déclin de l'influence du marxisme et 
des analyses matérialistes de la littérature d'un côté, et du triomphe de l'économie néoclassique (à l'origine d'une pratique toujours plus mathématisée et anhistorique de l'économie) de l'autre. Les contributions de The Fictions of American Capitalism appellent aujourd'hui à remettre en cause l'autonomisation de ces deux champs. Elles engagent les économistes à ouvrir leur discipline, à remédier à la fragmentation extrême des recherches dans laquelle elle a glissé et à voir ce qu'elle peut apprendre de la littérature. En retour, elles recommandent aux études littéraires de ne pas se laisser intimider par les catégories économiques, de participer à un questionnement général sur leur caractère naturel, et de ne pas céder au postulat que les œuvres littéraires existeraient abstraitement, en dehors de leurs conditions matérielles de production.

On peut souligner (suivant en cela les conseils méthodologiques des auteur-rices) que ce livre s'inscrit lui-même dans un contexte économique et politique particulier. Il s'agit tout d'abord de la crise économique et financière de 2008, qui est venue souligner les insuffisances de la vision anhistorique et décontextualisée promue par la théorie économique orthodoxe pour comprendre le capitalisme. Évoquée à de nombreuses reprises dans l'ouvrage, la «Grande Récession » constitue à la fois une crise financière et une crise de légitimité pour la science économique dominante, qui justifie que l'on regarde du côté de nouveaux outils - et en particulier de l'étude de la littérature - pour rendre compte des évolutions de l'économie. Le deuxième élément de contexte qui traverse ce livre est l'élection de Donald Trump à la présidence des États-Unis en 2016. Le président adepte de la " post-vérité » a plus que jamais rendu indispensable l'analyse des récits, de la fiction et de la concurrence des discours en particulier sur l'état de l'économie.

\section{Ce que le capitalisme doit à la fiction}

4 À travers des essais théoriques et des études de cas, le livre cherche à répondre aux deux questions évoquées plus haut, en évaluant les dettes réciproques de la fiction et $\mathrm{du}$ capitalisme états-unien. Une première série de contributions particulièrement originales s'intéresse ainsi aux fictions non-littéraires qui permettent à l'économie de fonctionner.

5 Ce sont en premier lieu les fictions (comme la monnaie ou le crédit) sur lesquelles sont fondées tout le système financier qui domine aujourd'hui le modèle capitaliste aux États-Unis. Ce sont également les mythes comme l'exceptionnalisme, ou l'absence totale de limite ("the boundless economy») d'une utopie états-unienne toujours recommencée, étudiée par Pierre Arnaud, qui servent de ciment idéologique au modèle capitaliste. Il s'agit aussi, dans les contributions de Robert Boyer ou de Jens Beckert, d'étudier les hypothèses (parfois fantaisistes) sur lesquelles les économistes professionnels s'appuient pour construire leurs modèles. Boyer comme Beckert soulignent le paradoxe qui fait que les économistes échafaudent des mondes parallèles, fictifs (qui ont parfois une valeur performative), mais ne s'intéressent pas au rôle très réel que joue la fiction dans les motivations des agents économiques. En insistant pour considérer ceux-ci comme "rationnels ", les économistes manquent l'importance des récits, de l'imagination, en particulier dans leur conception du futur.

6 L'ouvrage aborde encore la manière dont les entreprises, acteurs clés du capitalisme états-unien, s'appuient sur la mise en récit. Marie-Christine Pauwels, dans son étude de la pratique du storytelling depuis les années 1980 , met en évidence la manière dont les 
spécialistes du management aux États-Unis en sont venus à à soutenir le principe suivant: "fiction is more effective than arguments and hard evidence at getting messages across » (p. 113). La fiction devient alors un outil privilégié pour mobiliser des employés ou des consommateurs. De même, J.-H. Coste s'attache aux multiples récits de l'entreprenariat états-unien, des success stories aux ouvrages de conseils stratégiques, en passant par les textes académiques spécialisés, et montre ainsi la variété des narrations à l'œuvre pour construire un discours très influent à une période dominée par l'idéologie néo-libérale.

\section{Ce que la littérature doit au capitalisme}

7 Une deuxième série de textes propose des études d'œuvres littéraires afin de montrer ce qu'elles nous apprennent sur le capitalisme aux États-Unis. Au-delà d'un questionnement sur la littérature comme force critique ou complice du capitalisme, les contributeurs et contributrices s'interrogent sur la valeur épistémologique et heuristique des romans.

8 Certains chapitres s'intéressent pour cela à la manière dont les romanciers états-uniens ont $\mathrm{pu}$, depuis le $\mathrm{XIX}^{\mathrm{e}}$ siècle, se faire les vulgarisateurs de certaines théories économiques. Julia P. McLeod étudie en ce sens The Silent Partner d'Elizabeth Stuart Phelps (1870) comme une mise en récit littéraire des œuvres théoriques de l'économiste britannique John Stuart Mill, en particulier sur la liberté, les relations entre les classes sociales et le rôle économique des femmes. De même, Évelyne Payen-Variéras analyse les différentes théories des monopoles présentées dans The Octopus (1901) de Frank Norris, l'un des romans phares du Gilded Age sur l'emprise des grandes entreprises de chemin de fer sur la vie des fermiers états-uniens. Ces deux textes, comme celui de Vincent Dussol sur l'œuvre d'Ayn Rand (Atlas Shrugged, 1957, apologie du capitalisme débridé), insistent sur le succès seulement partiel de ces romans à servir de véhicules ou de porte-drapeaux à une théorie économique. Les uns comme les autres témoignent tout autant de l'état d'un débat économique à un moment donné que des hésitations et des contradictions dont ces théories sont porteuses.

En effet, si la littérature peut servir de lieu d'explicitation, d'outil permettant de maitriser des phénomènes économiques apparemment insaisissables, elle est aussi un espace de mise en scène de la complexité et des tensions qui sous-tendent ces phénomènes. Plusieurs contributions insistent notamment sur la manière dont la forme des romans, les choix esthétiques des auteurs et autrices peuvent faire écho à des moments de transition du capitalisme états-unien. Guillaume Tanguy recherche par exemple dans plusieurs romans de William Dean Howells les traces de l'évolution de l'auteur vis-à-vis des promesses de l'économie du Gilded Age. Jason Douglas, quant à lui, s'intéresse au Financier de Theodore Dreiser (1912) et à ce que l'œuvre traduit des incertitudes du capitalisme financier à l'orée du XXe siècle. En même temps qu'il met en scène des manœuvres financières complexes, Dreiser révèle aussi leur caractère mystérieux : "The economic problem that Dreiser describes is not the loss of [the hero's] money per se, but that he cannot understand why it happens or, what is worse, that perhaps there is no reason.» (p. 218). Pour J.-H. Coste, la narration polyphonique choisie par Philip Roth dans American Pastoral (1998) permet de retracer l'histoire d'un certain capitalisme industriel, d'une époque héroïque du début du $\mathrm{XX}^{\mathrm{e}}$ siècle jusqu'à son déclin à partir des années 1960. La littérature permet ici de faire coexister différents modes d'explication 
pour rendre compte d'évolutions complexes, sans rejeter les contradictions possibles ou les incertitudes. Les romans de Howells, Dreiser et Roth témoignent ainsi des efforts des auteurs pour négocier le passage d'une étape à une autre du capitalisme aux ÉtatsUnis.

Enfin, une dernière catégorie de textes s'attarde davantage sur le style des œuvres littéraires. William E. Dow décrit précisément la manière dont Richard Wright et Ann Perry, dans les années 1930 et 1940, ont mêlé journalisme et littérature afin de déconstruire les mécanismes d'un "capitalisme racialisé " (racialized capitalism) fonctionnant aux dépens des Afro-Américains. Bénédicte Chorier-Fryd s'intéresse quant à elle au motif récurrent de la décharge (Dumps) chez Thomas Pynchon, à la fois dans sa forme matérielle et virtuelle, comme lieu de résistance au capitalisme marchand (en particulier dans le récent Bleeding Edge, 2013). Sina Vatanpour enfin, fait le lien entre les romans de Paul Auster et de Martin Amis (City of Glass en 1985 et Money en 1984) dans lesquels les multiples jeux sur l'identité et l'intertextualité sont analysés comme autant d'échos à la perte de référent monétaire qui caractérise le capitalisme financier des années 1980.

\section{Plaidoyer pour les « humanités économiques »}

Il n'y a pas d'unité théorique absolue entre les différents chapitres du livre - certains tirent assez clairement du côté de la "New Economic Criticism» des années 1990, influencée par les cultural studies, quand d'autres s'en écartent franchement. Stephen Shapiro cherche dans son essai à relire l'histoire des genre littéraires états-uniens au prisme de catégories explicitement marxistes, quand Jean-Louis Brunel relit le style de William Gaddis avec les outils postmodernes de la «French theory ». Les directeurs de l'ouvrage, dans leur introduction, affirment s'appuyer sur la conception du capitalisme caractéristique de l'École de la Régulation (école théorique d'économistes français héritiers du marxisme et de la tradition institutionnaliste), sans qu'une telle filiation soit patente dans la totalité des chapitres. Cependant, l'introduction, comme la « coda » proposée par Peter Knight, s'attachent à recréer une unité, en appelant à rien de moins que la fondation d'une nouvelle discipline: les «humanités économiques » (economic humanities, p. 25 et p. 349 notamment). La proposition demande une plongée dans une réelle interdisciplinarité, qui convoque à la fois les études littéraires, l'économie, l'histoire des idées, la sociologie et l'anthropologie, « a daunting task » (p. 351), de l'aveu même des auteurs.

Cette nouvelle discipline reste encore à construire, mais The Fictions of American capitalism ouvre en tout cas des perspectives enthousiasmantes, et intéressera sans nul doute à la fois les spécialistes de l'économie états-unienne et les spécialistes d'études littéraires. Le champ couvert par les "humanités économiques » est potentiellement immense : comme le fait remarquer l'un des auteurs, «capitalism is a persistent leitmotif in American literature in general » (p. 282). 
INDEX

Thèmes : Recensions

\section{AUTEURS}

ALEXIA BLIN

MCF Civilisation des États-Unis, Sorbonne Nouvelle 